\title{
Red Blood Cell Distribution Width and Neutrophil-to-Lymphocyte Ratio in Predicting Adverse Outcomes of Acute Kidney Injury in Hospitalized Patients
}

\author{
Jiefu Zhu ${ }^{\mathrm{a}, \mathrm{b}}$ Cong Zeng ${ }^{\mathrm{c}}$ Lei Zhang ${ }^{\mathrm{a}}$ Shaoqun Shu ${ }^{\mathrm{a}}$ Yinghong Liu $^{\mathrm{a}}$ \\ Guochun Chen $^{a}$ Hong Liu $^{\mathrm{a}}$ Yu Liu $^{\mathrm{a}}$ Zheng Dong ${ }^{\mathrm{b}}$
}

aDepartment of Nephrology, The Second Xiangya Hospital at Central South University, Hunan Key Laboratory of Kidney Disease and Blood Purification, Changsha, China; ${ }^{b}$ Department of Cellular Biology and Anatomy, Medical College of Georgia at Augusta University and Charlie Norwood VA Medical Center, Augusta, GA, USA; 'Department of Nephrology, The Third Hospital of Changsha, Changsha, China

\section{Keywords}

Acute kidney injury · Red blood cell distribution width . Neutrophil-to-lymphocyte ratio $\cdot$ Complete blood cell count $\cdot$ Risk stratification

\begin{abstract}
Background: Acute kidney injury (AKI) is a common clinical condition with high morbidity and mortality. Early risk stratification by identifying patients at risk for death or dialysis requirement has important therapeutic implications for timely interventions. Objective: The aim of this study was to examine the association of routine blood test parameters, specifically red blood cell distribution width (RDW) and neutrophil-to-lymphocyte ratio (NLR), with the AKI patient outcomes. Methods: All adult patients hospitalized from January 1,2016, to June 30, 2016, in the Second Xiangya Hospital of Central South University were surveyed. Demographic characteristics, laboratory measurements, comorbidities, and outcomes of a total of 1,188 adult AKI patients were analyzed. Results: The incidence of AKI was $1.8 \%(1,188 / 65,329)$.
\end{abstract}

\begin{tabular}{ll}
\hline karger@karger.com & ( ) 2020 The Author(s) \\
Published by S. Karger AG, Basel & Karger \\
Targen access & This article is licensed under the Creative Commons Attribution- \\
NonCommercial-NoDerivatives 4.0 International License (CC BY- \\
NC-ND) (http://www.karger.com/Services/OpenAccessLicense). \\
Usage and distribution for commercial purposes as well as any dis- \\
tribution of modified material requires written permission.
\end{tabular}

The all-cause mortality was $16.0 \%(190 / 1,188)$. The multivariable relative risk of AKI mortality comparing high RDW with low RDW was 1.84 and the risk comparing high NLR with low NLR was 2.54. RDW and NLR combination showed additive values in stratifying high-risk patients, and the predictive power was comparable to the use of serum creatinine for staging AKI. In subgroup analyses, high RDW predicted prerenal AKI mortality better than intrinsic AKI. High RDW and NLR also independently predicted renal replacement therapy (RRT) requirement in AKI patients. In contrast, WBC count and platelet-to-lymphocyte ratio did not show obvious correlations with death and RRT requirement in AKI patients. Conclusion: The results support the potential usefulness of RDW and NLR in risk stratification of AKI patients, providing additional prognostic information for treatment and supportive care.

(c) 2020 The Author(s)

Published by S. Karger AG, Basel

Lei Zhang

Hunan Key Laboratory of Kidney Disease and Blood Purification

Department of Nephrology, The Second Xiangya Hospital, Central South University Changsha, Hunan 410011 (China)

zhanglxp@csu.edu.cn

Zheng Dong

Department of Cellular Biology and Anatomy

Medical College of Georgia and Charlie Norwood VA Medical Center

1459 Laney Walker Blvd, Augusta, GA 30912 (USA)

zdong@augusta.edu 


\section{Introduction}

Acute kidney injury (AKI) is common in hospitalized patients and affects $2-8 \%$ of all hospital admissions [1$3]$. Despite recent decades of research, AKI still results in high morbidity and mortality [4]. Early risk stratification has important implications in making timely intervention and determining the prognosis. Unfortunately, it is a clinical challenge to predict which patients will suffer AKI progression needing dialysis or proceeding to death. At present, novel serum and urinary kidney injury biomarkers, such as neutrophil gelatinase-associated lipocalin [5], angiotensinogen [6], and liver-type fatty acidbinding protein [7], have been proved to correlate to AKI progression and death. However, due to the heterogeneous and multifactorial nature of AKI, it seems unlikely a biomarker alone could achieve the accuracy like troponin in diagnosing acute myocardial infarction [8]. In addition, despite the promising potential of these biomarkers, their application is largely confined to research studies and difficult for widespread clinical use at present.

Inflammation and immune response are central components of the pathogenesis of AKI. Inflammation-associated parameters, like WBC count, red blood cell distribution width (RDW), neutrophil-to-lymphocyte ratio (NLR), and platelet-to-lymphocyte ratio (PLR), are routinely reported in complete blood cell count and available for most patients. Their implications in clinical outcome prediction have been explored in various diseases. $\mathrm{RDW}$ is a measurement of the change in circulating $\mathrm{RBC}$ volume and traditionally used for differentiating causes of anemia. Increased RDW, expressing greater heterogeneity in circulating erythrocytes size (anisocytosis), has been reported to strongly associate with the adverse outcomes in patients with chronic heart diseases [9]. NLR, a marker of host inflammation calculated by dividing the counts of neutrophils by lymphocytes, may have the potential to predict survival in a variety of human cancers [10]. Although RDW has been reported to correlate with declining renal function and adverse outcomes in patients with chronic kidney disease $[11,12]$ and AKI patients $[13,14]$, no studies have systematically investigated the predictive ability of routine blood test parameters in AKI patients. Therefore, our study examined the possible correlation of blood cell changes and the outcomes of inhospital AKI patients, in order to determine the prognostic value of blood cell parameters for early risk stratification in AKI patients.

\section{Materials and Methods}

\section{Patient Cohort}

The study cohort screened all patients aged 16-99 years admitted in the Second Xiangya Hospital of Central South University, a regional tertiary hospital in southern China, between January 1, 2016 , and June 30, 2016. A total of 65, 329 admissions with hospitalization for $>24 \mathrm{~h}$ were investigated. AKI patients were identified according to the Kidney Disease: Improving Global Outcomes guidelines in 2012 except urine output criteria for lack of the records of the urine volume in most hospitalized patients. Patients who had maintenance renal replacement therapy (RRT), ESRD, nephrectomy, solitary kidney, kidney transplantation, or baseline serum $\mathrm{Cr}$ (SCr) levels less than $40 \mu \mathrm{mol} / \mathrm{L}$ were excluded. Twenty AKI patients with hematological malignancy were excluded due to their abnormalities in blood cells.

\section{Data Collection}

Demographic data, clinical concomitant diseases (determined by the diagnosis codes at admission and discharge), and biochemical data were all collected from electronic hospitalization databases. Parameters in a routine blood test were collected at admission. AKI was defined as an increase in SCr of more than 26.5 $\mu \mathrm{mol} / \mathrm{L}$ within $48 \mathrm{~h}$ or a confirmed/speculated increase of SCr of 1.5 -fold over baseline with 7 days. The AKI stage was determined by the highest SCr level after AKI development according to Kidney Disease: Improving Global Outcomes guidelines except urine output criteria; stage 1 , increase of $\geq 26.5 \mu \mathrm{mol} / \mathrm{L}$ within $48 \mathrm{~h}$ or increase of SCr to $\geq 1.5$ times baseline, which was known or presumed to have occurred within 7 days; stage 2, increase of SCr to 2.0-2.9 times from baseline; stage 3, increase of SCr to 3.0 times from baseline or to $\geq 353.6 \mu \mathrm{mol} / \mathrm{L}$, or RRT requirement. AKI etiology, classification, and stage were diagnosed by 2 nephrologists. NLR was calculated as the ratio of neutrophil number divided by lymphocyte number and PLR was the ratio of platelet count divided by lymphocyte count. CKD was determined based on the diagnostic coding, history of renal injury more than 3 months prior to admission, or an estimated glomerular filtration rate $<60 \mathrm{~mL} /$ $\mathrm{min} / 1.73 \mathrm{~m}^{2}$ for more than 3 months. The outcome of AKI patients was determined according to hospital records and follow-ups. For the patients discharged alive or not on dialysis, we followed up by reviewing medical records or telephone assessment to the subject or family members at 1 and 2 months after enrollment.

\section{Statistical Analysis}

We used SPSS 22.0 software for statistical analysis. A $p$ value $<0.05$ was regarded statistically significant. The non-normal distribution variables were presented as median (interquartile range), the normal distribution data were expressed as the mean \pm SD for continuous variables, and the categorical variables were presented as $n(\%)$, as appropriate. The comparison between groups was measured by $t$ test for continuous variables in normal distribution, the Kruskal-Wallis test for variables in non-normal distribution, and the $\chi^{2}$ test for categorical variables. We conducted univariate and multivariate logistic regression analysis to determine the risk factors for clinical outcomes. For multivariate analysis, we adjusted age, gender, heart disease, hypertension, cerebrovascular disease, CKD, chronic obstructive pulmonary disease, chronic liver disease, diabetes, cancer, hemoglobin, hematocrit, serum albumin, total bilirubin, and serum uric acid with or without AKI classifica- 
Fig. 1. Flowchart of the study cohort selection. SCr, serum creatinine; AKI, acute kidney injury; KDIGO, Kidney Disease: Improving Global Outcomes; RRT, renal replacement therapy.

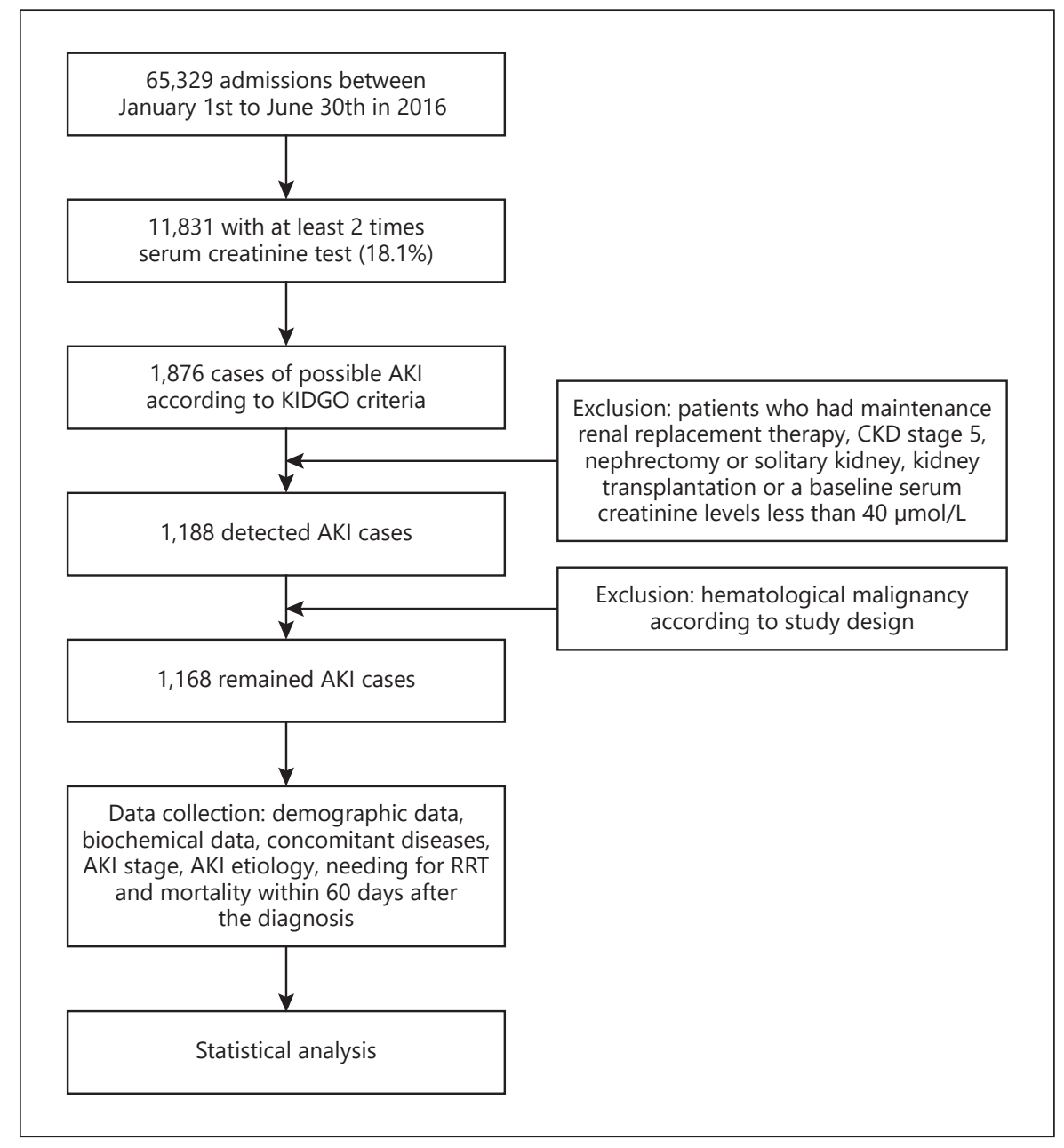

tion. The results were presented as the odds ratio (OR) and its $95 \%$ confidence interval (CI). The receiver operating characteristic (ROC) curve was used to evaluate the predictive value of individual biomarker to the 60-day mortality and determine the cutoff value of RDW and NLR to predict AKI death.

\section{Results}

\section{Baseline Characteristics of AKI Patients}

Of the 65,329 hospital admissions in the study between January 1 and June 30 in 2016, 11,831 admissions had at least 2 repeated SCr tests, which identified 1,188 patients with AKI, indicating an incidence rate of $1.8 \%$. The allcause 60-day mortality in AKI patients was $16.0 \%$ $(190 / 1,188)$. A total of 1,168 AKI patients were further analyzed following the exclusion of 20 patients with hematological malignancy due to their remarkable abnormalities in blood cells (Fig. 1); 184 patients (15.4\%) died within 60 days after AKI diagnosis. The baseline characteristics of AKI patients are shown in Table 1. Patients in AKI stage 1, stage 2, or stage 3 accounted for 63.3, 20.5, and $16.2 \%$, respectively. Prerenal and intrinsic-renal insults were the predominant causes of AKI with their proportions being 55.3 and $36.3 \%$, respectively. Compared with survivors, the non-survivors tended to be older and had increased prevalence of cerebrovascular disease, diabetes, and heart disease. The non-survivors had remarkably higher rates for requiring the therapy for RRT. Notably, the 2 groups of patients had no statistical differences in the prevalence of CKD and hypertension or the value of WBC and serum uric acid.

\section{Comparison of Characteristics according to RDW/NLR Binaries}

The characteristics of patients divided according to RDW and NLR binaries are presented in Table 2. The optimal RDW cutoff value as $13.95 \%$ and NLR cutoff value 
Table 1. Patients' characteristics

\begin{tabular}{|c|c|c|c|c|}
\hline Variable & $\begin{array}{l}\text { ALL } \\
(n=1,168)\end{array}$ & $\begin{array}{l}\text { Survivors } \\
(n=984)\end{array}$ & $\begin{array}{l}\text { Non-survivors } \\
(n=184)\end{array}$ & $p$ value \\
\hline Age, years & $58(47,67)$ & $56(46,66)$ & $64(50,75)$ & 0.000 \\
\hline Male & $766(65.6 \%)$ & $648(65.9 \%)$ & $118(64.1 \%)$ & 0.673 \\
\hline \multicolumn{5}{|l|}{ Underlying disease } \\
\hline Cerebrovascular disease & $130(11.1 \%)$ & $93(9.5 \%)$ & $37(20.1 \%)$ & 0.000 \\
\hline Diabetes & $184(15.8 \%)$ & $139(14.1 \%)$ & $45(24.5 \%)$ & 0.000 \\
\hline Hypertension & $422(36.1 \%)$ & $349(35.5 \%)$ & $73(39.7 \%)$ & 0.278 \\
\hline Heart disease & $405(34.7 \%)$ & $324(32.9 \%)$ & $81(44.0 \%)$ & 0.000 \\
\hline Cancer & $245(20.9 \%)$ & $205(20.8 \%)$ & $40(21.7 \%)$ & 0.768 \\
\hline Chronic liver disease & $142(12.2 \%)$ & $111(11.3 \%)$ & $31(16.8 \%)$ & 0.037 \\
\hline CKD & $466(39.9 \%)$ & $396(40.2 \%)$ & $70(38 \%)$ & 0.623 \\
\hline COPD & $49(4.2 \%)$ & $35(3.6 \%)$ & $14(7.6 \%)$ & 0.025 \\
\hline \multicolumn{5}{|l|}{ AKI stage } \\
\hline 1 & $739(63.3 \%)$ & $663(67.4 \%)$ & $76(41.3 \%)$ & 0.000 \\
\hline 2 & $240(20.5 \%)$ & $189(19.2 \%)$ & $51(27.7 \%)$ & \\
\hline 3 & $189(16.2 \%)$ & $132(13.4 \%)$ & $57(31.0 \%)$ & \\
\hline \multicolumn{5}{|l|}{ Cause of AKI } \\
\hline Prerenal & $646(55.3 \%)$ & $539(54.8 \%)$ & $107(58.2 \%)$ & 0.000 \\
\hline Intrinsic-renal & $425(36.3 \%)$ & $349(35.5 \%)$ & $76(41.3 \%)$ & \\
\hline Post-renal & $50(4.28 \%)$ & $49(5.0 \%)$ & $1(0.5 \%)$ & \\
\hline Unclassified & $47(4.02 \%)$ & $47(4.8 \%)$ & 0 & \\
\hline RRT requirement & $99(8.5 \%)$ & $49(5.0 \%)$ & $50(27.2 \%)$ & 0.000 \\
\hline \multicolumn{5}{|l|}{ Laboratory index } \\
\hline WBC $\left(10^{9} / \mathrm{L}\right)$ & $7.5(5.6,11.2)$ & $7.4(5.6,10.7)$ & $7.6(5.0,12.6)$ & 0.105 \\
\hline Platelet counts $\left(10^{9} / \mathrm{L}\right)$ & $179.0(123.0,238.0)$ & $183.0(128.0,242.7)$ & $163.5(100.0,219.0)$ & 0.000 \\
\hline Albumin, $\mathrm{g} / \mathrm{L}$ & $33.0(28.5,37.5)$ & $33.5(29,37.9)$ & $30.3(26.9,35.4)$ & 0.000 \\
\hline Total bilirubin, $\mu \mathrm{mol} / \mathrm{L}$ & $11.8(7.6,21.8)$ & $11.6(7.5,20.9)$ & $13.0(8.6,28.2)$ & 0.025 \\
\hline Serum uric acid, $\mu \mathrm{mol} / \mathrm{L}$ & $348.7(257.8,452.8)$ & $348.9(260.9,445.7)$ & $327(225.2,490.5)$ & 0.401 \\
\hline Hemoglobin, $g / \mathrm{L}$ & $116.0(97.0,133.0)$ & $118.0(99.0,135.0)$ & $108.5(89.8,127.0)$ & 0.000 \\
\hline RDW, \% & $13.7(12.9,15.2)$ & $13.6(12.8,14.9)$ & $14.7(13.6,16.5)$ & 0.000 \\
\hline Hematocrit, \% & $35.4(29.7,40.1)$ & $35.9(30.7,40.4)$ & $33.3(27.3,38.4)$ & 0.000 \\
\hline PLR & $146.0(99.2,224.6)$ & $142.4(100.0,218.6)$ & $173.6(92.0,256.6)$ & 0.045 \\
\hline NLR & $4.7(2.5,9.3)$ & $4.4(2.3,8.6)$ & $6.8(3.7,13.2)$ & 0.000 \\
\hline
\end{tabular}

AKI, acute kidney injury; COPD, chronic obstructive pulmonary disease; CKD, chronic kidney disease; RRT, renal replacement therapy; RDW, red blood cell distribution width; NLR, neutrophil-to-lymphocyte ratio; PLR, platelet-to-lymphocyte ratio.

as 5.51 were based on the ROC curve analysis for AKI death (Table 5). Notably, the patients with high level of RDW or NLR were generally older and had higher prevalence of chronic obstructive pulmonary disease, higher AKI stage, and higher mortality and RRT requirement rates than the patients with low level of RDW or NLR. The prevalence of chronic liver disease and heart disease increased with high level of RDW. Patients with high level of NLR showed a higher rate of cerebrovascular disease and hypertension, but a lower rate of cancer, compared with patients in low NLR.

\section{Prognostic Significance in All-Cause AKI Mortality}

The level of RDW or NLR was associated with mortality in AKI patients (Table 3). The risk of death in AKI patients with high RDW or NLR was 1.84 (95\% CI, 1.202.81) and 2.54 (95\% CI, 1.74-3.72), respectively, compared to the patients with low RDW or NLR. As RDW or NLR alone was an independent risk predictive factor of mortality in AKI patients and did not correlate with each other $(r=0.04, p>0.05)$ (Table 7), we hypothesized that the combination of RDW and NLR may have extra prognostic values. We further categorized patients into 3 groups as (1) RDW-low and NLR-low, (2) RDW-high or NLR-high, and (3) RDW-high and NLR-high. This sim- 
Table 2. Epidemiological and clinical characteristics of AKI patients according to RDW and NLR

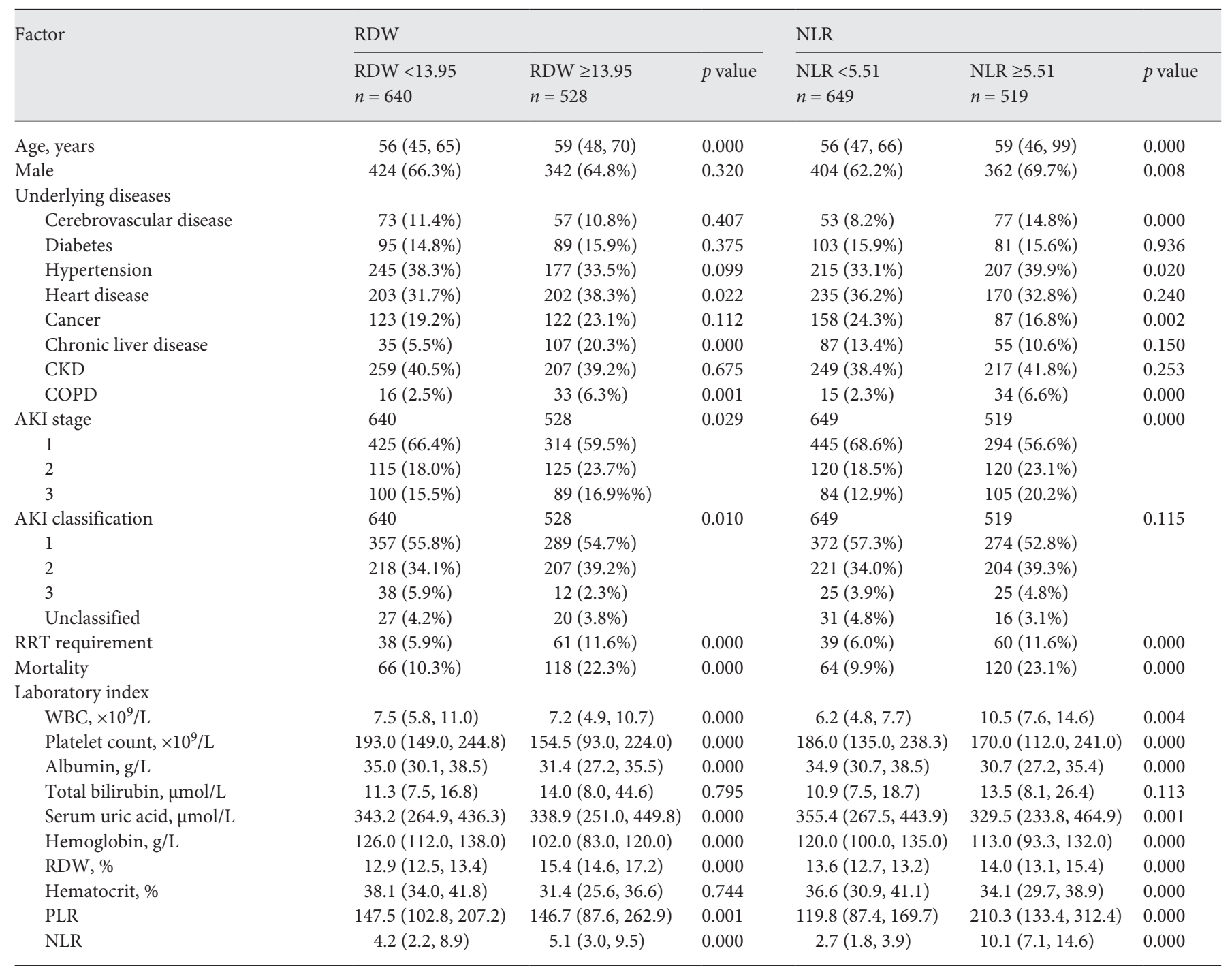

$\mathrm{AKI}$, acute kidney injury; COPD, chronic obstructive pulmonary disease; CKD, chronic kidney disease; RRT, renal replacement therapy; RDW, red blood cell distribution width; NLR, neutrophil-to-lymphocyte ratio; PLR, platelet-to-lymphocyte ratio.

ple and effective way succeeded in stratifying patients into low-, intermediate (OR, 3.04; 95\% CI, 1.76-5.25)-, and high-risk (OR, 5.61; 95\% CI, 3.11-10.12) groups. Notably, the predicting power of RDW and NLR for AKI mortality was comparable to the use of SCr-based AKI staging (AKI stage 2: OR, 1.97; stage 3: OR, 5.23). A nonlinear relationship between WBC and PLR with all-cause mortality was observed in AKI patients. Therefore, we categorized patients into 3 groups according to $\mathrm{WBC}$ and PLR values. The group with the highest tertile of WBC counts had greater mortality risks (OR, 1.84; 95\% CI, 1.17-2.91) than that of intermediate WBC counts after adjustment. Similar to the effects of WBC, the lowest AKI mortality was seen in the patients with the mid-portion of PLR range (Table 3).

\section{Prognostic Significance in Prerenal and \\ Intrinsic-Renal Mortality}

There was a strong correlation between NLR and mortality risk in both prerenal AKI and intrinsic-renal AKI patients (Table 4). Compared to patients in the low NLR group, patients in the high NLR group suffered 2.55 and 2.78-fold mortality risk in prerenal AKI and intrinsic-renal AKI, respectively (Table 4). However, high RDW functioned as a stronger predictor of prerenal AKI deaths than intrinsic AKI deaths. For intrinsic-renal AKI pa- 
Table 3. Univariate and multivariate logistic regression analysis of inflammatory indicators and AKI stage to predict AKI mortality

\begin{tabular}{|c|c|c|c|c|}
\hline \multirow[t]{2}{*}{ Variable } & \multicolumn{2}{|l|}{ Unadjusted } & \multicolumn{2}{|l|}{ Adjusted } \\
\hline & OR (95\% CI) & $p$ value & OR (95\% CI) & $p$ value \\
\hline \multicolumn{5}{|l|}{ RDW } \\
\hline Low $(<13.95 \%)$ & 1 (reference) & & 1 (reference) & \\
\hline High ( $\geq 13.95 \%)$ & $2.50(2.22-5.07)$ & 0.000 & $1.84(1.20-2.81)$ & 0.005 \\
\hline \multicolumn{5}{|l|}{ NLR } \\
\hline Low $(<5.51)$ & 1 (reference) & & 1 (reference) & \\
\hline $\operatorname{High}(\geq 5.51)$ & $2.75(2.100-5.194)$ & 0.000 & $2.54(1.74-3.72)$ & 0.000 \\
\hline \multicolumn{5}{|l|}{$\mathrm{WBC}$} \\
\hline Low $\left(<6.26 \times 10^{9} / \mathrm{L}\right)$ & $1.20(0.80-1.78)$ & 0.010 & $1.37(0.88-2.15)$ & 0.163 \\
\hline Intermediate $\left(6.26-9.78 \times 10^{9} / \mathrm{L}\right)$ & 1 (reference) & & 1 (reference) & \\
\hline $\operatorname{High}\left(>9.78 \times 10^{9} / \mathrm{L}\right)$ & $1.62(1.10-2.39)$ & 0.015 & $1.84(1.17-2.91)$ & 0.009 \\
\hline \multicolumn{5}{|l|}{ PLR } \\
\hline Low $(<112)$ & $1.27(0.84-1.92)$ & 0.252 & $1.25(0.78-1.99)$ & 0.350 \\
\hline Intermediate (112-195) & 1 (reference) & & 1 (reference) & \\
\hline $\operatorname{High}(>195)$ & $1.84(1.25-2.71)$ & 0.002 & $1.73(1.12-2.66)$ & 0.002 \\
\hline \multicolumn{5}{|l|}{ Combined RDW + NLR } \\
\hline RDW-low + NLR-low & 1 (reference) & & 1 (reference) & \\
\hline RDW-high or NLR-high & $3.39(2.06-5.56)$ & 0.000 & $3.04(1.76-5.25)$ & 0.000 \\
\hline RDW-high + NLR-high & $6.99(4.17-11.70)$ & 0.000 & $5.61(3.11-10.12)$ & 0.000 \\
\hline \multicolumn{5}{|l|}{ AKI stage } \\
\hline 1 & 1 (reference) & & 1 (reference) & \\
\hline 2 & $2.35(1.59-3.48)$ & 0.000 & $1.97(1.26-3.08)$ & 0.000 \\
\hline 3 & $3.77(2.55-5.57)$ & 0.000 & $5.23(3.28-8.33)$ & 0.000 \\
\hline \multicolumn{5}{|l|}{ RRT } \\
\hline Non-requirement & 1 (reference) & 0.000 & 1 (reference) & 0.000 \\
\hline Requirement & $7.12(4.62-10.99)$ & & $8.65(5.00-15.00)$ & \\
\hline
\end{tabular}

RDW, NLR, PLR, combined RDW + NLR, and AKI stage were adjusted separately in models that included age, gender, heart disease, hypertension, cerebrovascular disease, chronic kidney disease, chronic obstructive pulmonary disease, chronic liver disease, type 2 diabetes, cancer, AKI classification, hemoglobin, hematocrit, total bilirubin, and serum uric acid (multivariable analysis model 1). AKI, acute kidney injury; RDW, red blood cell distribution width; RRT, renal replacement therapy; NLR, neutrophil-to-lymphocyte ratio; PLR, platelet-tolymphocyte ratio; $\mathrm{OR}$, odds ratio; $\mathrm{CI}$, confidence interval.

tients, the mortality risk of high versus low RDW was not statistically significant after adjustment. However, in prerenal AKI patients, the risk of death was about 2-fold in participants with higher RDW values compared to those with lower RDW levels (Table 4), and furthermore the death risk was nearly 6-fold in AKI patients with "RDWhigh and NLR-high" compared to that in those with "RDW-low and NLR-low" (Table 4). These results indicated that RDW levels could better predict mortality risk in prerenal AKI than intrinsic-renal AKI.

\section{Ability for Predicting AKI Patient Death}

We further analyzed the prognostic abilities of blood cell parameters in predicting AKI death in 60 days by ROC curve. The area under the ROC curve (AUC) of
AKI death for RDW was 0.63 (95\% CI, 0.59-0.68), and RDW level higher than the optimal cutoff value of $13.95 \%$ predicted AKI mortality with a sensitivity of $64.1 \%$ and specificity of $58.3 \%$ (Table 5; Fig. 2a). The RDW cutoff value was similar to that of another study on AKI patents in coronary care unit, which determined $14.0 \%$ as the optimal cutoff value [14]. NLR has an AUC of 0.65 (95\% CI, 0.60-0.69) and the best cutoff value of 5.51 with a sensitivity of $63.5 \%$ and a specificity of $61.0 \%$. The value of AUC indicated RDW or NLR is similar to serum albumin (0.62) and hemoglobin (0.59) in predicting AKI mortality. However, the AUC of WBC or PLR was not statistically significant compared to 0.5 (Table 5; Fig. 2a). 
Table 4. Univariate and multivariate logistic regression analysis of RDW and NLR to predict prerenal and intrinsic-renal AKI mortality

\begin{tabular}{|c|c|c|c|c|}
\hline \multirow[t]{2}{*}{ Variable } & \multicolumn{2}{|l|}{ Unadjusted } & \multicolumn{2}{|l|}{ Adjusted } \\
\hline & OR $(95 \% \mathrm{CI})$ & $p$ value & OR $(95 \%$ CI $)$ & $p$ value \\
\hline \multicolumn{5}{|l|}{ Prerenal AKI } \\
\hline \multicolumn{5}{|l|}{ RDW } \\
\hline Low $(<13.95)$ & 1 (reference) & & 1 (reference) & \\
\hline High $(\geq 13.95)$ & $2.76(1.79-4.27)$ & 0.000 & $2.13(1.24-3.73)$ & 0.008 \\
\hline \multicolumn{5}{|l|}{ NLR } \\
\hline Low $(<5.51)$ & 1 (reference) & & 1 (reference) & \\
\hline $\operatorname{High}(\geq 5.51)$ & $2.56(1.67-3.93)$ & 0.000 & $2.55(1.54-4.23)$ & 0.000 \\
\hline \multicolumn{5}{|l|}{ Combined RDW + NLR } \\
\hline RDW-low + NLR-low & 1 (reference) & & 1 (reference) & \\
\hline RDW-high or NLR-high & $3.45(1.83-6.51)$ & 0.000 & $3.05(1.46-6.38)$ & 0.003 \\
\hline RDW-high + NLR-high & $7.08(3.63-13.81)$ & 0.000 & $6.03(2.66-13.66)$ & 0.000 \\
\hline \multicolumn{5}{|l|}{ Intrinsic-renal AKI } \\
\hline \multicolumn{5}{|l|}{ RDW } \\
\hline Low $(<13.95)$ & 1 (reference) & & 1 (reference) & \\
\hline High $(\geq 13.95)$ & $2.05(1.23-3.42)$ & 0.006 & $1.48(0.74-2.92)$ & 0.275 \\
\hline \multicolumn{5}{|l|}{ NLR } \\
\hline Low $(<5.51)$ & 1 (reference) & & 1 (reference) & \\
\hline High $(\geq 5.51)$ & $3.26(1.90-5.58)$ & 0.000 & $2.78(1.50-5.21)$ & 0.001 \\
\hline \multicolumn{5}{|l|}{ Combined RDW + NLR } \\
\hline RDW-low + NLR-low & 1 (reference) & & 1 (reference) & \\
\hline RDW-high or NLR-high & $3.97(1.71-9.23)$ & 0.001 & $2.86(1.09-7.54)$ & 0.034 \\
\hline RDW-high + NLR-high & $7.13(3.01-16.93)$ & 0.000 & $4.96(1.77-13.90)$ & 0.002 \\
\hline
\end{tabular}

RDW, NLR, and combined RDW + NLR were adjusted separately in models that included age, gender, heart disease, hypertension, cerebrovascular disease, chronic kidney disease, chronic obstructive pulmonary disease, chronic liver disease, type 2 diabetes, cancer, hemoglobin, hematocrit, hemoglobin, total bilirubin, and serum uric acid. AKI, acute kidney injury; RDW, red blood cell distribution width; NLR, neutrophil-to-lymphocyte ratio; $\mathrm{OR}$, odds ratio; $\mathrm{CI}$, confidence interval.

Table 5. ROC analysis of blood cell parameters in predicting AKI death

\begin{tabular}{llcll}
\hline Biomarker & AUC $(95 \% \mathrm{CI})$ & Best cutoff value & Sensitivity, \% & Specificity, \% \\
\hline RDW, \% & $0.63(0.59-0.68)$ & 13.95 & 64.1 & 58.3 \\
NLR & $0.65(0.60-0.69)$ & 5.51 & 63.5 & 61.0 \\
WBC, $\times 10^{9} / \mathrm{L}$ & $0.54(0.49-0.59)$ & 13.75 & 25.0 & 87.2 \\
PLR & $0.55(0.50-0.60)$ & 162.4 & 55.4 & 58.4 \\
Serum albumin, g/L & $0.62(0.57-0.66)$ & 30.85 & 66.3 & 54.8 \\
Hemoglobin, g/L & $0.59(0.55-0.64)$ & 102.5 & 71.8 & 54.1 \\
\hline
\end{tabular}

AUC, area under the ROC curve; AKI, acute kidney injury; RDW, red blood cell distribution width; NLR, neutrophil-to-lymphocyte ratio; PLR, platelet-to-lymphocyte ratio; CI, confidence interval.

\section{Prognostic Significance in RTT Requirement}

The univariate and multivariable association of blood cell parameters in predicting RRT requirement in the entire cohort is demonstrated in Table 6. Patients with high
RDW level had higher risk odds of requiring RRT (OR, 1.99; 95\% CI, 1.13-3.50) than patients with low RDW. NLR also predicted the AKI progression to RRT requirement (OR, 1.65; 95\% CI, 1.01-2.71). The combination of 
Table 6. Univariate and multivariate logistic regression analysis of inflammatory indicators in CBC to predict the need for RRT

\begin{tabular}{|c|c|c|c|c|}
\hline \multirow[t]{2}{*}{ Variable } & \multicolumn{2}{|l|}{ Unadjusted } & \multicolumn{2}{|l|}{ Adjusted } \\
\hline & OR $(95 \% \mathrm{CI})$ & $p$ value & OR $(95 \% \mathrm{CI})$ & $p$ value \\
\hline \multicolumn{5}{|l|}{ RDW } \\
\hline Low $(<13.95 \%)$ & 1 (reference) & & 1 (reference) & \\
\hline High $(\geq 13.95 \%)$ & $2.07(1.36-3.16)$ & 0.001 & $1.99(1.13-3.50)$ & 0.017 \\
\hline \multicolumn{5}{|l|}{ NLR } \\
\hline Low $(<5.51)$ & 1 (reference) & & 1 (reference) & \\
\hline $\operatorname{High}(\geq 5.51)$ & $2.05(1.34-3.16)$ & 0.001 & $1.65(1.01-2.71)$ & 0.046 \\
\hline \multicolumn{5}{|l|}{ WBC } \\
\hline Low $\left(<6.26 \times 10^{9} / \mathrm{L}\right)$ & $1.06(0.58-1.93)$ & 0.849 & $1.137(0.57-2.26)$ & 0.714 \\
\hline Intermediate $\left(6.26-9.78 \times 10^{9} / \mathrm{L}\right)$ & 1 (reference) & & 1 (reference) & \\
\hline High $\left(>9.78 \times 10^{9} / \mathrm{L}\right)$ & $1.55(0.99-2.44)$ & 0.056 & $1.09(0.64-1.85)$ & 0.762 \\
\hline \multicolumn{5}{|l|}{ PLR } \\
\hline Low $(<112)$ & $1.03(0.61-1.73)$ & 0.924 & $0.86(0.45-1.62)$ & 0.452 \\
\hline Intermediate (112-195) & 1 (reference) & & 1 (reference) & \\
\hline High (>195) & $1.32(0.80-2.17)$ & 0.276 & $1.27(0.73-2.21)$ & 0.725 \\
\hline \multicolumn{5}{|l|}{ Combined RDW + NLR } \\
\hline RDW-low + NLR-low & 1 (reference) & & 1 (reference) & \\
\hline RDW-high or NLR-high & $2.10(1.17-3.78)$ & 0.013 & $1.97(0.98-3.96)$ & 0.059 \\
\hline RDW-high + NRL-high & $3.92(2.13-7.20)$ & 0.000 & $3.18(1.47-6.86)$ & 0.003 \\
\hline
\end{tabular}

RDW, NLR, PLR, and combined RDW + NLR were adjusted separately in models that included age, gender, heart disease, hypertension, cerebrovascular disease, chronic kidney disease, chronic obstructive pulmonary disease, chronic liver disease, diabetes, cancer, AKI classification, hemoglobin, hematocrit, total bilirubin, and serum uric acid (multivariable analysis model 1). RDW, red blood cell distribution width; NLR, neutrophil-tolymphocyte ratio; PLR, platelet-to-lymphocyte ratio; OR, odds ratio; CI, confidence interval.

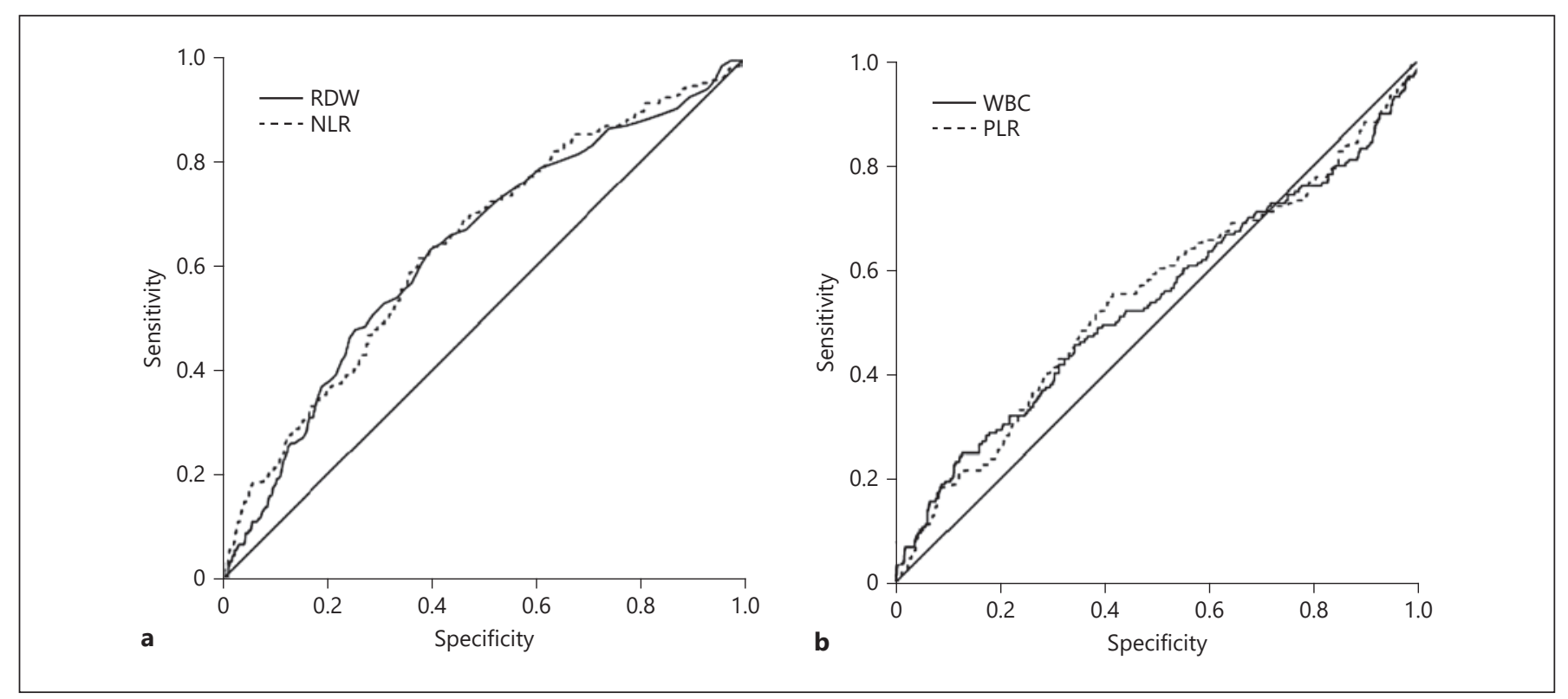

Fig. 2. ROC analysis of blood cell parameters in predicting AKI mortality. a ROC curves of RDW and NLR in predicting 60-day AKI mortality. b ROC curves of WBC and PLR in predicting 60-day AKI mortality. ROC, receiver operating characteristic; AKI, acute kidney injury; RDW, red blood cell distribution width; NLR, neutrophil-to-lymphocyte ratio; PLR, platelet-to-lymphocyte ratio. 
RDW and NLR further increased the ability of risk stratification. The prognostic ability of WBC and PLR was much weaker than that of RDW and NLR.

\section{Discussion}

In this study, we have demonstrated that RDW or NLR may function as an independent risk factor for dialysis requirement and all-cause mortality in hospitalized AKI patients. Patients with RDW $>13.95 \%$ denoted 2 -fold risk of dialysis requirement and 1.8 -fold risk of death, compared with patients with low RDW after adjusting multiple potential confounding factors. Patients with NLR $>5.51$ suffered 1.7 -fold risk of dialysis requirement and 2.5 -fold risk of death. These results support that RDW and NLR, 2 parameters in routine blood test, may provide additional prognostic information for the risk prediction of AKI.

AKI has become a huge medical and economic burden worldwide [4]. In China, a recent nationwide study involving 44 hospitals indicated the all-cause in-hospital mortality was $12.4 \%$ in AKI patients [3]. Consistent with these previous studies on Chinese population, we found the all-cause 60 -day AKI mortality was $16.0 \%$ in the Second Xiangya Hospital located in southern China, indicating a high mortality rate in AKI. Because of the great burden and adverse outcome of AKI, stratifying high-risk patients and identifying useful biomarkers that allow early intervention are very important. Recent prospective multicenter studies reported that several systemic inflammation-associated biomarkers, like IL-6 [15], IL-18 [16], IL-8 [17], and soluble urokinase plasminogen activator receptor [18], showed better performance in predicting adverse outcomes than kidney injury biomarkers such as kidney injury molecule 1 and neutrophil gelatinase-associated lipocalin [17]. While recognizing the importance of inflammatory markers, in our retrospective study, all patients had routine blood tests but only a very small percentage of them had measurements of inflammatory markers like IL-6 and C-reactive protein. This actually supports the significance of identifying inflammatory indicators in routine blood test for risk stratification.

Among routinely reported inflammatory parameters in complete blood cell count, we found RDW and NLR outperformed WBC and PLR in predicting AKI progression requiring dialysis and patient death. RDW significantly correlated with hemoglobin; however, the predictive ability of RDW was independent of hemoglobin and even superior to hemoglobin in AUC. Though both RDW and
Table 7. Correlation coefficient $(r)$ between baseline RDW/NLR and selected parameters

\begin{tabular}{lrrrr}
\hline Variable & $\begin{array}{l}\text { RDW } \\
(r)\end{array}$ & $p$ value & $\begin{array}{l}\text { NLR } \\
(r)\end{array}$ & $p$ value \\
& 0.02 & 0.444 & 0.07 & 0.011 \\
\hline Age (years) & & & & \\
Comorbidities & -0.09 & 0.004 & 0.02 & 0.373 \\
$\quad$ Hypertension & -0.03 & 0.393 & 0.10 & 0.000 \\
Cerebrovascular disease & 0.09 & 0.002 & -0.09 & 0.002 \\
Cancer & 0.31 & 0.000 & -0.04 & 0.220 \\
Chronic liver diseases & & & & \\
Laboratory parameters & 1 & - & 0.04 & 0.144 \\
$\quad$ RDW (\%) & 0.04 & 0.144 & 1 & - \\
$\quad$ NLR & -0.46 & 0.000 & -0.06 & 0.063 \\
Hemoglobin (g/L) & -0.44 & 0.000 & -0.08 & 0.011 \\
Hematocrit $(\%)$ & 0.07 & 0.013 & 0.34 & 0.000 \\
WBC $\left(\times 10^{9} / \mathrm{L}\right)$ & 0.01 & 0.844 & 0.58 & 0.000 \\
PLR & -0.17 & 0.000 & -0.20 & 0.000 \\
Albumin $(\mathrm{g} / \mathrm{L})$ & 0.34 & 0.000 & 0.02 & 0.609 \\
$\quad$ Total bilirubin $(\mu \mathrm{mol} / \mathrm{L})$ & & & & \\
\hline
\end{tabular}

RDW, red blood cell distribution width; NLR, neutrophil-tolymphocyte ratio; PLR, platelet-to-lymphocyte ratio.

NLR may reflect systemic inflammation, they did not correlate with each other $(r=0.04, p>0.05)$ (Table 7). Therefore, the method of combining RDW and NLR succeeded in stratifying AKI patients into low-, intermediate-, and high-risk groups. The combination of RDW and NLR was comparable to the use of SCr for AKI stage in predicting AKI patient death, suggesting a promising potential of combining RDW and NLR in AKI risk stratification.

Both prerenal and intrinsic-renal AKI reflect an abrupt decrease in kidney function, but they have a distinct pathophysiology. The high RDW level was more strongly associated with the mortality risk in prerenal AKI than intrinsic-renal AKI. The reasons are currently unclear. The association between elevated RDW level and the progression of cardiovascular diseases has been described in previous studies $[9,19]$, and indeed, patients with elevated RDW level more likely have cardiovascular diseases in our study. Aggravated cardiovascular diseases may lead to failure of the general circulation and a decrease in renal perfusion pressure, which could cause prerenal AKI (like cardiorenal syndrome). On the other hand, intrinsic-renal AKI is often caused by renal intrinsic ischemia and nephrotoxic medications, where an association of RDW with those disorders is rarely reported.

The exact pathophysiological mechanism underlying the association of high RDW with adverse outcomes is not well understood. Elevated RDW results from en- 
trance of immature erythrocytes into the circulation, which is caused by fluctuation of erythropoiesis. Growing evidence suggests that inflammatory cytokines and poor nutritional status may alter iron metabolism, suppress erythropoietin production, and enhance erythropoietin resistance $[20,21]$. Indeed, in our study, patients with higher RDW levels (>13.95\%) were generally older and suffered from hypoalbuminemia and anemia, indicating systemic inflammation and malnutrition may enhance RDW level in AKI patients. Therefore, RDW may be a parameter reflecting chronic inflammatory and poor nutritional status. Besides, high RDW itself may be a potential mediator of the poor clinical outcome since its influence on AKI death is independent of age, albumin, and hemoglobin. Further studies are needed to clarify potential confounding factors and the detailed association between RDW and AKI adverse outcomes.

There are also several limitations to this study. First, this is a retrospective, single-center study, where some of the potential confounding factors like inflammatory cytokines were not measured in most of the patients. Second, AKI was diagnosed only according to SCr changes and did not include urine output. Last, we did not perform a longer follow-up on AKI patients to evaluate the risk factors that affected their long-term prognosis.

In summary, this study investigated the potential of routine blood test parameters for predicting adverse outcomes of AKI, including dialysis requirement and mortality. Our results support the independent predictive power of RDW and NLR in general AKI patients. These inexpensive and routinely reported indicators may provide valuable information in early risk stratification of AKI patients.

\section{Acknowledgements}

We sincerely thank the patients who participated in this study and the medical staff who took care of the patients at the Second Xiangya Hospital, Central South University.

\section{Statement of Ethics}

The research was approved by the Ethics Committee of the Second Xiangya Hospital at Central South University (protocol reference number 2013-050-01). Written informed consent was not required because of the nonintervention and retrospective chart review design.

\section{Disclosure Statement}

The authors have declared that no conflict of interest exists.

\section{Funding Sources}

The work was supported partly by the grants from the National Key R\&D Program of China (2018YFC1312700) and the National Natural Science Foundation of China.

\section{Author Contributions}

J.Z., L.Z., C.Z., and Z.D. designed this study; carried out the collection, analysis, and interpretation of data; and drafted the manuscript. S.S. helped in the collection of data of patients. J.Z., L.Z., G.C., and Z.D. revised the manuscript. Y.L., H.L., and Y.L. provided good suggestions for study design and data collection.

\section{References}

1 Chertow GM, Burdick E, Honour M, Bonventre JV, Bates DW. Acute kidney injury, mortality, length of stay, and costs in hospitalized patients. J Am Soc Nephrol. 2005 Nov; 16(11):3365-70.

2 Xu X, Nie S, Liu Z, Chen C, Xu G, Zha Y, et al. Epidemiology and clinical correlates of AKI in Chinese hospitalized adults. Clin J Am Soc Nephrol. 2015 Sep 4;10(9):1510-8.

3 Yang L, Xing G, Wang L, Wu Y, Li S, Xu G, et al. Acute kidney injury in China: a cross-sectional survey. Lancet. 2015 Oct 10; 386(10002):1465-71.

4 Lameire NH, Bagga A, Cruz D, De Maeseneer J, Endre Z, Kellum JA, et al. Acute kidney injury: an increasing global concern. Lancet. 2013 Jul 13;382(9887):170-9.
5 Nickolas TL, Schmidt-Ott KM, Canetta P, Forster C, Singer E, Sise M, et al. Diagnostic and prognostic stratification in the emergency department using urinary biomarkers of nephron damage: a multicenter prospective cohort study. J Am Coll Cardiol. 2012 Jan 17; 59(3):246-55.

6 Chen C, Yang X, Lei Y, Zha Y, Liu H, Ma C, et al. Urinary biomarkers at the time of AKI diagnosis as predictors of progression of AKI among patients with acute cardiorenal syndrome. Clin J Am Soc Nephrol. 2016 Sep 7; 11(9):1536-44

7 Ferguson MA, Vaidya VS, Waikar SS, Collings FB, Sunderland KE, Gioules CJ, et al. Urinary liver-type fatty acid-binding protein predicts adverse outcomes in acute kidney injury. Kidney Int. 2010 Apr;77(8):708-14.
8 Alge JL, Arthur JM. Biomarkers of AKI: a review of mechanistic relevance and potential therapeutic implications. Clin J Am Soc Nephrol. 2015 Jan 7;10(1):147-55.

9 Patel KV, Ferrucci L, Ershler WB, Longo DL, Guralnik JM. Red blood cell distribution width and the risk of death in middle-aged and older adults. Arch Intern Med. 2009 Mar 9;169(5):515-23.

10 Mei Z, Shi L, Wang B, Yang J, Xiao Z, Du P, et al. Prognostic role of pretreatment blood neutrophil-to-lymphocyte ratio in advanced cancer survivors: a systematic review and meta-analysis of 66 cohort studies. Cancer Treat Rev. 2017 Jul;58:1-13. 
11 Sicaja M, Pehar M, Derek L, Starcevic B, Vuletic V, Romic Z, et al. Red blood cell distribution width as a prognostic marker of mortality in patients on chronic dialysis: a single center, prospective longitudinal study. Croat Med J. 2013 Feb;54(1):25-32.

12 Vashistha T, Streja E, Molnar MZ, Rhee CM, Moradi H, Soohoo M, et al. Red cell distribution width and mortality in hemodialysis patients. Am J Kidney Dis. 2016 Jul;68(1):11021.

13 Oh HJ, Park JT, Kim JK, Yoo DE, Kim SJ, Han $\mathrm{SH}$, et al. Red blood cell distribution width is an independent predictor of mortality in acute kidney injury patients treated with continuous renal replacement therapy. Nephrol Dial Transplant. 2012 Feb;27(2):589-94.
$14 \mathrm{Hu}$ Y, Liu H, Fu S, Wan J, Li X. Red blood cell distribution width is an independent predictor of AKI and mortality in patients in the coronary care unit. Kidney Blood Press Res. 2017 Dec 8;42(6):1193-204.

15 Zhang WR, Garg AX, Coca SG, Devereaux PI, Eikelboom J, Kavsak P, et al. Plasma IL-6 and IL-10 concentrations predict AKI and longterm mortality in adults after cardiac surgery. J Am Soc Nephrol. 2015 Dec;26(12):3123-32.

16 Pike F, Murugan R, Keener C, Palevsky PM, Vijayan A, Unruh M, et al. Biomarker enhanced risk prediction for adverse outcomes in critically ill patients receiving RRT. Clin J Am Soc Nephrol. 2015 Aug 7;10(8):1332-9.

17 Greenberg JH, Zappitelli M, Jia Y, ThiessenPhilbrook HR, de Fontnouvelle CA, Wilson FP, et al. Biomarkers of AKI progression after pediatric cardiac surgery. J Am Soc Nephrol. 2018 Feb 22;29(5):1549-56.
18 Hayek SS, Leaf DE, Samman Tahhan A, Raad M, Sharma S, Waikar SS, et al. Soluble urokinase receptor and acute kidney injury. $\mathrm{N}$ Engl J Med. 2020 Jan 30;382(5):416-26.

19 Dabbah S, Hammerman H, Markiewicz W, Aronson D. Relation between red cell distribution width and clinical outcomes after acute myocardial infarction. Am J Cardiol. 2010 Feb 1;105(3):312-7.

20 Weiss G, Goodnough LT. Anemia of chronic disease. N Engl J Med. 2005 Mar 10; 352(10):1011-23.

21 Förhécz Z, Gombos T, Borgulya G, Pozsonyi Z, Prohászka Z, Jánoskuti L. Red cell distribution width in heart failure: prediction of clinical events and relationship with markers of ineffective erythropoiesis, inflammation, renal function, and nutritional state. Am Heart J. 2009 Oct;158(4):659-66. 